\title{
FLUJO INTERNO MIXTO CON DIFUSIÓN DOBLE DE CALOR Y MASA EN UNA CAVIDAD RECTANGULAR
}

\section{MIXED INTERNAL FLOW WITH DOUBLE DIFFUSION OF HEAT AND MASS}

\author{
Carlos H. Salinas Lira ${ }^{1} \quad$ Yerko A. Gatica Lagos ${ }^{2} \quad$ Pedro A. Pacheco Fernandoy ${ }^{3}$ \\ Recibido 31 de octubre de 2007, aceptado 28 de mayo de 2008 \\ Received: October 31, 2007 Accepted: May 28, 2008
}

\begin{abstract}
RESUMEN
El presente trabajo muestra resultados de transporte de calor y masa (doble) al interior de una cavidad rectangular ocasionado por un flujo convectivo forzado y natural (mixto). El modelo matemático consta de las ecuaciones de Navier-Stokes para describir el flujo laminar de un fluido incompresible. Además es modelado el transporte de calor y masa a través de dos ecuaciones diferenciales lineales de segunda orden, incorporando en forma lineal los efectos de los gradientes de concentración y de temperaturas. El modelo matemático es resuelto numéricamente a través del Método de Volúmenes Finitos descrito en coordenadas curvilíneas y variables colocalizadas, representando los términos difusivos a través de diferencia central y usando un esquema potencial para los términos convectivos. Se muestran resultados originales de patrones de flujo: Isotermas, isoconcentraciones y números de Nusselt y de Sherwood en regiones características para el problema de flujo interno mixto con difusión de calor y masa.
\end{abstract}

Palabras clave: Difusión, convección, cavidad rectangular, flujo laminar, simulación.

\begin{abstract}
The present work shows the relationship of the transport of heat and mass (double) in a rectangular cavity caused by a forced and natural convection. The mathematical model consists of the Navier-Stokes equations to describe the incompressible laminar flow. The transport of heat and mass are modelled through two second order no-linear differential equations, incorporating in linear way the effects of gradients of concentrations and temperature. The mathematical problems is solved numerically through the Finite Volumes Method, in curvilinear coordinated and co-located variables, representing the diffusion through central difference and using a potential schemes for the convection terms. Original results of flow models: Isotherms, isoconcentrations and numbers of Nusselt and Sherwood in characteristic regions for the mixed internal flow problem with heat and mass are shown.
\end{abstract}

Keywords: Diffusion, convection, square cavity, laminar flow, simulation.

\section{INTRODUCCIÓN}

El presente trabajo plantea un estudio numérico para caracterizar conjuntamente el desarrollo de un flujo interno del tipo mixto conjugado con transporte de calor y masa en una cavidad rectangular.

Diversos autores han abordado la modelación numérica trabajando con cavidades rectangulares. En [1] se desarrollan aplicaciones donde se considera a la temperatura dependiente de la conductividad y la viscosidad. Otros investigadores han desarrollado modelos capaces de calcular la convección libre sobre un medio poroso en una cavidad inclinada [2]. Ahora estudios que están más en la línea del presente trabajo analizan el efecto de flujo convectivo interno en el ámbito de la refrigeración de componentes electrónicos [3].

Diversos modelos que contemplan la variación de concentración y temperatura han sido publicados en la literatura, como por ejemplo aquellos en los cuales se describe la convección doble en una cavidad [4-7]. Los

\footnotetext{
1 Departamento de Ingeniería Mecánica. Universidad del Bío-Bío. Av. Collao 1202. Fono: 731455/731499. Fax: 731022. Concepción, Chile. E-mail: Casali@ubiobio.cl

2 Departamento de Ingeniería Mecánica. Universidad del Bío-Bío. Av. Collao 1202. Fono: 731455/731499. Fax: 731022. Concepción, Chile. E-mail:ygatica@ubiobio.cl

3 Departamento de Mecânica. COPPE-UFRJ. Rio de Janeiro, Brasil. E-mail: ppachecofernandoy@yahoo.es
} 
modelos muestran una relación inversa entre el número de Darcy y el ángulo de inclinación de la cavidad. En particular, en [8] se realizan comparaciones para distintos números adimensionales y configuraciones de condiciones de contorno. Este estudio es la base a partir de la cual se desarrolla el presente trabajo.

Más recientemente, se presentan estudios sobre convección externa sobre cilindros [9] como también trabajos en el área de nanotecnología [10], en medios porosos [11-12] y exploraciones tridimensionales [13].

La metodología usada en el presente trabajo tiene un enfoque de análisis numérico validado por resultados de problemas similares disponible en la literatura especializa. Primero, el programa es validado con resultados de difusión doble presentados en [8] y, segundo, por resultados de convección mixta interna presentados por [4]. Lo anterior permite plantear el análisis y mostrar resultados originales consistentes del problema conjugado de flujo interno mixto con difusión doble.

La modelación para el presente trabajo se basa en las ecuaciones de Navier-Stokes para describir el flujo laminar de un fluido incompresible, acoplando linealmente los efectos de los gradientes de concentración y de temperatura. El modelo matemático es resuelto numéricamente a través del Método de Volúmenes Finitos [14] descrito en coordenadas curvilíneas [15-16] y variables colocalizadas, representando los términos difusivos a través de diferencia central y usando un esquema potencial para los términos convectivos. En términos generales, la formulación numérica fue realizada según es descrita por Maliska [17] y las mallas son generadas en base a una ecuación Poisson [15]. Se validan los algoritmos para dos problemas asintóticos: Difusión doble y Flujo interno mixto. Luego se muestran diversos resultados que caracterizan al problema Flujo Interno Mixto Acoplado con Difusión Doble de Calor y Masa, objeto del presente trabajo.

\section{MODELO FÍSICO}

El problema físico en estudio corresponde a uno de convección mixta con difusión doble de calor y masa esquematizado en la figura 1. En esta se puede observar una cámara rectangular, de altura $\mathrm{H}$ y ancho L. En términos generales, las paredes verticales son impermeables con temperaturas constantes $\mathrm{T}_{\mathrm{h}} \mathrm{y} \mathrm{T}_{\mathrm{c}}$ y las horizontales son consideradas adiabáticas con concentraciones $\mathrm{C}_{h}$ y $\mathrm{C}_{\mathrm{l}}$. Además existe ingreso (velocidad uniforme $\mathrm{V}$ ) y salida de masa en una sección de altura $\mathrm{d}$ en las paredes verticales. Dependiendo de los valores en los contornos se pueden tener problemas asintóticos ampliamente difundidos en la literatura especializada:
Convección natural $\left(\mathrm{V}=\mathrm{C}_{\mathrm{h}}=\mathrm{C}_{1}=0\right)$, convección forzada $\left(\mathrm{T}_{\mathrm{h}}=\mathrm{T}_{\mathrm{c}}=\mathrm{C}_{\mathrm{h}}=\mathrm{C}_{1}=0\right)$, convección mixta $\left(\mathrm{C}_{\mathrm{h}}=\mathrm{C}_{1}=0\right) \mathrm{y}$ Difusión doble $(\mathrm{V}=0)$.

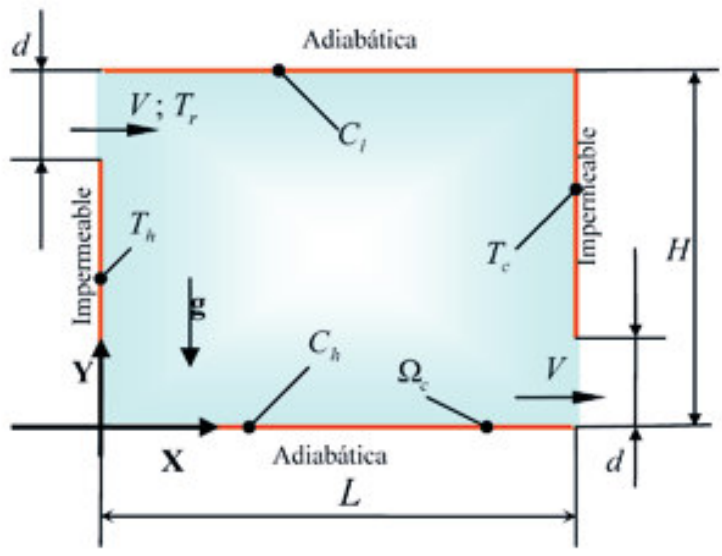

Figura 1. Esquema del problema.

\section{MODELO MATEMÁTICO}

\section{Dominio fluido}

El modelo matemático considera las ecuaciones de continuidad, momentum (Navier-Stokes), energía y masa para un fluido incompresible de densidad $\rho$, viscosidad dinámica $\mu$, conductividad $\mathrm{k}$, difusividad $\mathrm{D}$ y afectado por la fuerza gravitacional g como mostrado en las ecuaciones (1-4).

$$
\begin{gathered}
\nabla(\mathbf{u})=0 \\
\rho\left[\frac{\partial \mathbf{u}}{\partial t}+\mathbf{u}(\nabla \mathbf{u})\right]=-\nabla p+\nabla(\mu \nabla \mathbf{u})+\mathbf{F}_{\rho} \\
\rho c_{p}\left[\frac{\partial T}{\partial t}+\mathbf{u}(\nabla T)\right]=\nabla(k \nabla T)+\dot{n}_{T} \\
\frac{\partial \rho_{A}}{\partial t}+\mathbf{u}\left(\nabla \rho_{A}\right)=\nabla\left(D_{A-B} \nabla \rho_{A}\right)+\dot{n}_{A}
\end{gathered}
$$

Donde $T$ representa la temperatura $[\mathrm{K}], \rho_{A}$ la densidad de la especie transportada $\mathrm{A}\left[\mathrm{kg} / \mathrm{m}^{3}\right], c_{p}$ calor específico $[\mathrm{J} / \mathrm{kg} \mathrm{K}], k$ conductividad $[\mathrm{W} / \mathrm{m} \mathrm{K}], D_{A-B}$ coeficiente de difusión de masa A en el medio B $\left[\mathrm{m}^{2} / \mathrm{s}\right], \dot{n}_{T}$, fuente de energía $\left[\mathrm{W} / \mathrm{m}^{3}\right]$ y $\dot{n}_{A}$ fuente de masa $\left[\mathrm{kg} / \mathrm{s} \mathrm{m}^{3}\right]$.

En este contexto, las fuerzas de empuje debido a la variación de densidad, considerando efectos de expansión térmica 
y de gradiente de concentraciones, son expresadas por la formulación de Oberdeck-Boussinesq:

$$
\mathbf{F}_{\rho}=\rho \mathbf{g}\left[\beta_{T}\left(T-T_{0}\right)+\beta_{S}\left(\rho_{A}-\rho_{A_{0}}\right)\right]
$$

Donde $\rho_{A o}$ densidad de referencia $\left[\mathrm{kg} / \mathrm{m}^{3}\right], T_{0}$ temperatura de referencia $[\mathrm{K}], \beta_{T}$ coeficiente de expansión térmica $[1 / \mathrm{K}], \beta_{s}$ coeficiente de expansión por variación de concentración $\left[\mathrm{m}^{3} / \mathrm{kg}\right]$ y $\mathbf{g}$ aceleración de gravedad $\left[\mathrm{m} / \mathrm{s}^{2}\right]$.

Para el caso bidimensional, el modelo expresado por las ecuaciones (1) a (5) para un flujo transitorio bidimensional $(\mathbf{u}=(u, v))$ en el plano XY (Y orientado en $-\mathbf{g})$ de un fluido incompresible ( $\rho=$ cte.), se puede escribir de la siguiente forma:

$$
\begin{gathered}
\frac{\partial u}{\partial x}+\frac{\partial v}{\partial y}=0 \\
\rho\left[\frac{\partial u}{\partial t}+\left(u \frac{\partial u}{\partial x}+v \frac{\partial u}{\partial y}\right)\right]=-\frac{\partial p}{\partial x}+\mu\left[\frac{\partial}{\partial x}\left(\frac{\partial u}{\partial x}\right)+\frac{\partial}{\partial y}\left(\frac{\partial u}{\partial y}\right)\right]
\end{gathered}
$$

$\rho\left[\frac{\partial v}{\partial t}+\left(u \frac{\partial v}{\partial x}+v \frac{\partial v}{\partial y}\right)\right]=-\frac{\partial p}{\partial y}+\mu\left[\frac{\partial}{\partial x}\left(\frac{\partial v}{\partial x}\right)+\frac{\partial}{\partial y}\left(\frac{\partial v}{\partial y}\right)\right]$

$$
+\rho g\left[\beta_{T}\left(T-T_{0}\right)+\beta_{S}\left(\rho_{A}-\rho_{A_{0}}\right)\right]
$$

$$
\rho c_{p}\left[\frac{\partial T}{\partial t}+u \frac{\partial T}{\partial x}+v \frac{\partial T}{\partial y}\right]=\frac{\partial}{\partial x}\left(k \frac{\partial T}{\partial x}\right)+\frac{\partial}{\partial y}\left(k \frac{\partial T}{\partial y}\right)+\dot{n}_{T}
$$

$$
\frac{\partial \rho_{A}}{\partial t}+u \frac{\partial \rho_{A}}{\partial x}+v \frac{\partial \rho_{A}}{\partial y}=\frac{\partial}{\partial x}\left(D_{A-B} \frac{\partial \rho_{A}}{\partial x}\right)+\frac{\partial}{\partial y}\left(D_{A-B} \frac{\partial \rho_{A}}{\partial y}\right)+\dot{n}_{A}
$$

\section{Formulación genérica}

Las ecuaciones (6) a (10) pueden ser expresadas genéricamente por (11), cuyos coeficientes para cada ecuación de transporte son mostrados en la tabla 1.

$$
\begin{aligned}
& \frac{\partial\left(c_{\phi} \phi\right)}{\partial t}+\frac{\partial(\rho u \phi)}{\partial x}+\frac{\partial(\rho v \phi)}{\partial y} \\
& =\frac{\partial p}{\partial y}+\frac{\partial}{\partial x}\left(a_{d} \frac{\partial \phi}{\partial x}\right)+\frac{\partial}{\partial y}\left(a_{d} \frac{\partial \phi}{\partial y}\right)+a_{m} \phi+F
\end{aligned}
$$

Tabla 1. Coeficientes de la ecuación (17).

\begin{tabular}{|c|c|c|c|c|}
\hline$\phi$ & $\mathbf{c}_{\phi}$ & $\mathbf{a}_{\mathbf{d}}$ & $\mathbf{F}$ & Ecuación \\
\hline 1 & 0 & 0 & 0 & continuidad \\
\hline $\mathrm{u}$ & $\rho$ & $\mu$ & 0 & momentum en $X$ \\
\hline$v$ & $\rho$ & $\mu$ & $\rho g\left[\begin{array}{l}\beta_{T}\left(T-T_{0}\right) \\
+\beta_{S}\left(\rho_{A}-\rho_{A_{0}}\right)\end{array}\right]$ & momentum en $Y$ \\
\hline$T$ & $\rho c_{p}$ & $k$ & 0 & Energía \\
\hline$\rho_{A}$ & 1 & $D_{A-B}$ & 0 & Masa \\
\hline
\end{tabular}

\section{Condiciones de contorno}

Las condiciones iniciales son las siguientes:

$$
\left.\begin{array}{l}
(u, v)=(0,0) \\
\begin{array}{l}
\rho_{A}=0 \\
T=T_{c}
\end{array}
\end{array}\right\} \text { en } \forall \quad \text { para } t=0
$$

En cuanto a las condiciones de contorno en el dominio fluido finito considerado, son las siguientes:

$$
\begin{aligned}
& (u, v)= \begin{cases}(0,0) & \Omega_{\mathrm{e}} \\
(v, 0) & \text { en } \mathrm{x}=0 \text { con } \mathrm{H}-\mathrm{d} \leq \mathrm{y} \leq H\end{cases} \\
& T=\left\{\begin{array}{lll}
T_{h} & \text { en } \mathrm{x}=0 \\
T_{c} & \text { en } & \mathrm{x}=\mathrm{L} \\
\mathrm{T}_{\mathrm{f}} & \text { en } \mathrm{x}=0 \text { con } \mathrm{H}-\mathrm{d} \leq \mathrm{y} \leq H
\end{array}\right. \\
& \frac{\partial \mathrm{T}}{\partial \mathrm{y}}=0 \text { en } \begin{array}{ll}
\mathrm{y}=0 \\
\mathrm{y}=\mathrm{H}
\end{array} \\
& \rho_{A}=\left\{\begin{array}{ll}
\rho_{h} & \text { en } \mathrm{y}=0 \\
\rho_{c} & \text { en } \quad \mathrm{y}=\mathrm{H} \\
\rho_{\mathrm{f}} & \text { en } \mathrm{x}=0
\end{array} \text { con } \mathrm{H}-\mathrm{d} \leq \mathrm{y} \leq H\right. \\
& \frac{\partial \rho_{A}}{\partial \mathrm{y}}=0 \text { en } \quad \begin{array}{l}
\mathrm{x}=0 \\
\mathrm{x}=\mathrm{L}
\end{array}
\end{aligned}
$$

El modelo matemático es resuelto numéricamente a través del Método de Volúmenes Finitos descrito en coordenadas curvilíneas y variables colocalizadas, representando los términos difusivos a través de diferencia central y usando un esquema potencial para los términos convectivos como el descrito en [17]. 


\section{RESULTADOS}

\section{Difusión doble}

Con el objeto de validar el modelo se generan resultados para el caso de difusión doble, lo cual implica no considerar ingreso y egreso de masa ( $\mathrm{V}=0$ ). Resultados obtenidos para determinados adimensionales definidos a continuación para valores dados en la tabla 2 , considerando mallas de $15 \times 30$ a 40x80, son mostrados en las figuras (2-6).

$$
\begin{aligned}
& \operatorname{Pr}=\frac{\rho c_{p} V}{k} \\
& G \mathrm{r}_{T}=\frac{g \beta_{T}\left[T_{h}-T_{c}\right] H^{3}}{v^{2}} \\
& S c=\frac{v}{D_{A-B}}=\operatorname{Pr} \cdot L e \\
& L e=\frac{\alpha}{D_{A-B}} \\
& N=\frac{\beta_{S} \Delta \rho_{A}}{\beta_{T} \Delta T} \\
& D a=\frac{K}{H^{2}} \\
& \operatorname{Re}=\frac{V H}{v} \\
& R i=\frac{G r}{\operatorname{Re}^{2}} \\
& S h=\frac{h_{l} H}{D_{A-B}} \\
& N u=\frac{h H}{k} \\
& \mathrm{~A}=\frac{\mathrm{L}}{\mathrm{H}} \\
& \theta=\frac{T-T_{c}}{T_{h}-T_{c}} \\
& C^{*}=\frac{C-C_{l}}{C_{h}-C_{l}} \\
& t^{*}=\frac{t}{H^{2} / v} \\
& U=\frac{u}{v / H} ; \quad V=\frac{v}{v / H} \\
& X=\frac{x}{H} \quad ; \quad Y=\frac{y}{H} \quad \text { Longitudes }
\end{aligned}
$$

Tabla 2. Adimensionales para difusión doble.

\begin{tabular}{|l|l|l|l|l|}
\hline $\mathbf{N}$ & $\mathbf{P r}$ & $\mathbf{G r}_{\mathbf{T}}$ & $\mathbf{L e}$ & $\mathbf{A}$ \\
\hline 2.5 & 7 & $10^{4}$ & 100 & 2 \\
\hline
\end{tabular}

La figura 2 presenta las mallas no uniformes del tipo cosenoidad utilizadas. La figura 3 muestra el análisis de consistencia con relación al tipo de malla y la figura 4 de forma similar para el paso de integración. Las figuras 5 y 6 muestran evoluciones transitorias en forma de líneas de corriente, isotermas y isoconcentraciones respectivamente. En términos generales, se puede concordar que con las mallas de 30x60 y pasos de tiempo 50 [s] se obtienen resultados convergentes. En cuanto a la cualidad de los resultados, estos resultaron ser similares a los obtenidos por Bennacer [8].
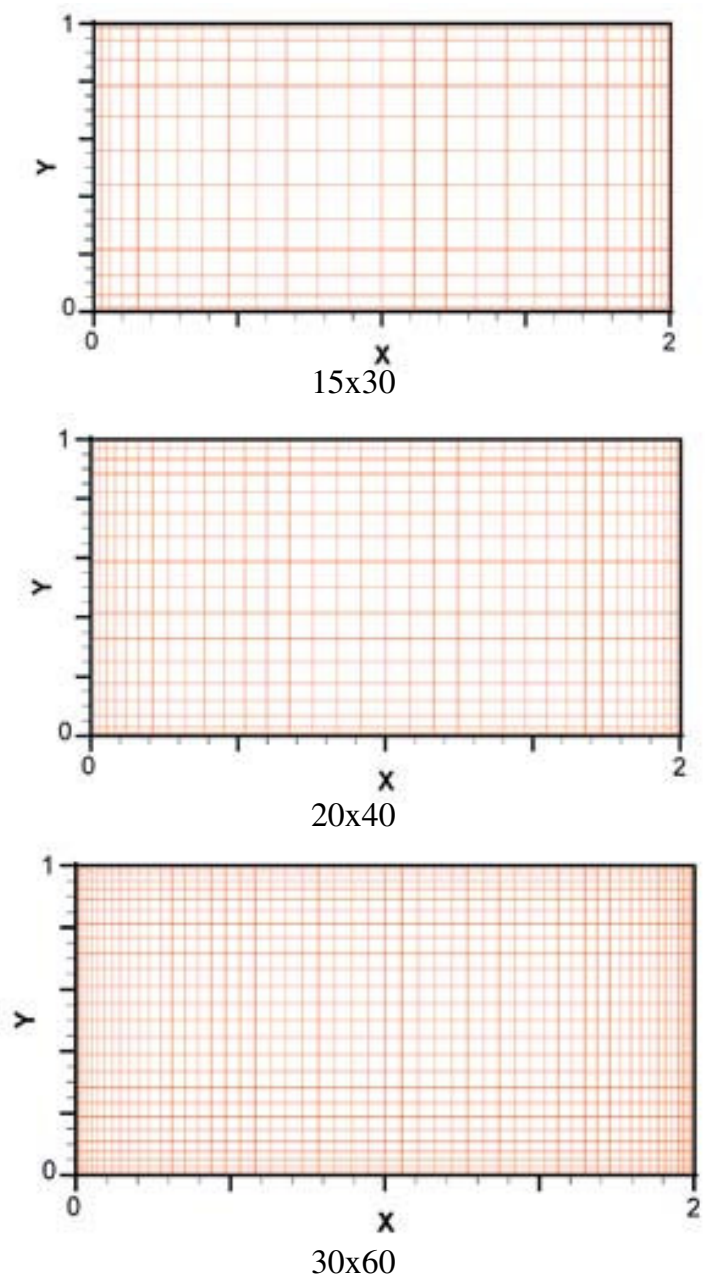

Figura 2. Mallas utilizadas en difusión doble. (Continúa) 


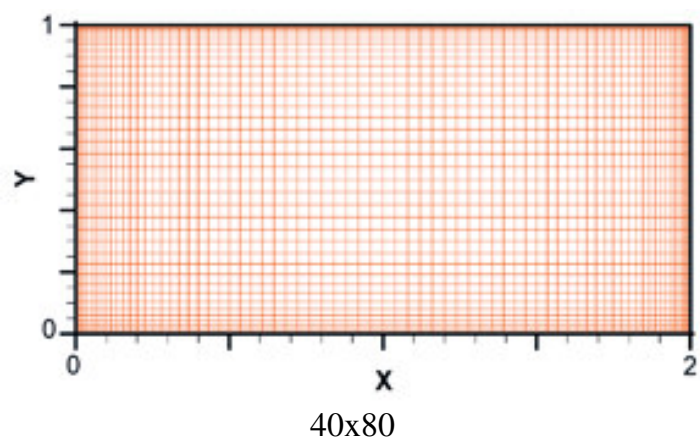

Figura 2. Mallas utilizadas en difusión doble.

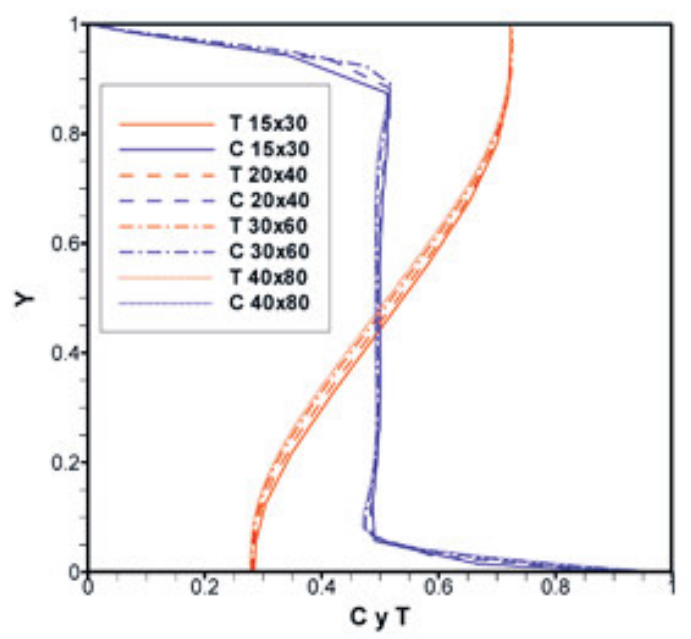

Figura 3. Análisis de convergencia de temperatura y concentración en la sección $\mathrm{x}=\mathrm{L} / 2$ en función del tipo de malla.

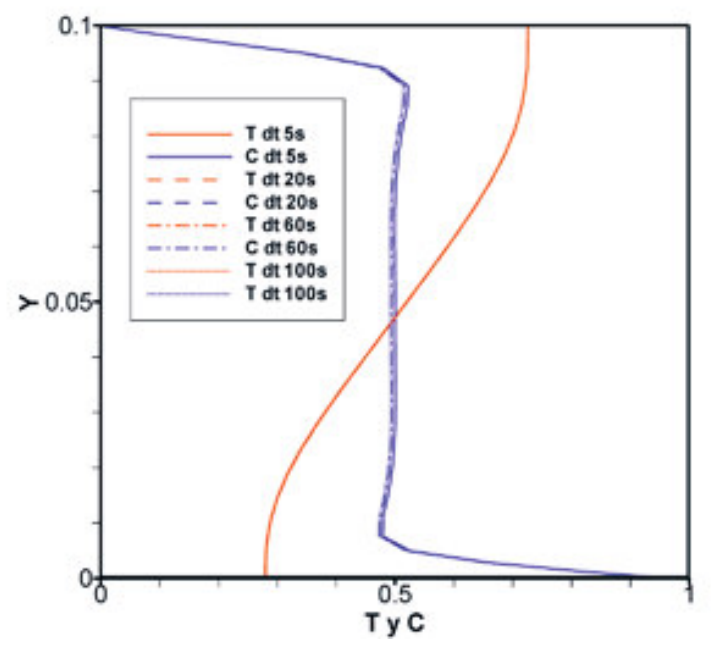

Figura 4. Análisis de convergencia de temperatura y concentración en la sección $\mathrm{x}=\mathrm{L} / 2$ (Malla $30 x 60$ y $5<\mathrm{dt}<100$ [s]).

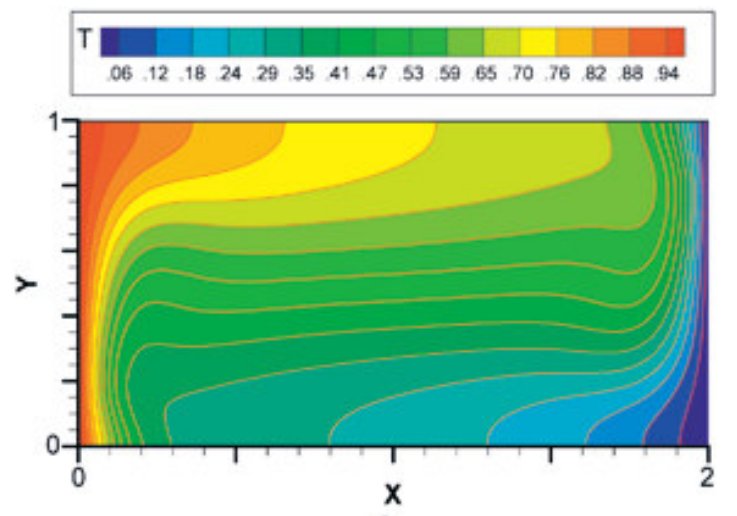

a)

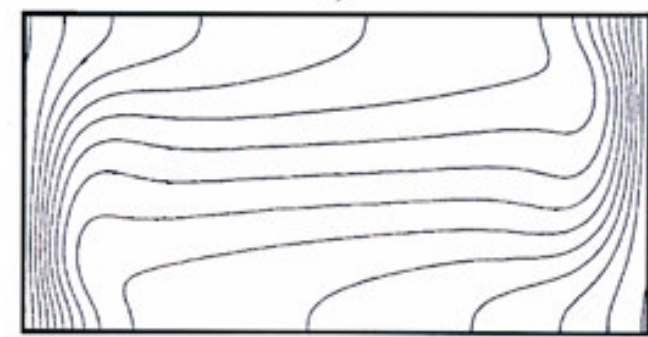

b)

Figura 5. Distribución de isotermas: a) Presente trabajo: malla 30x60; $\mathrm{t}=50 \mathrm{y} \mathrm{b}$ ) Bennacer [8].

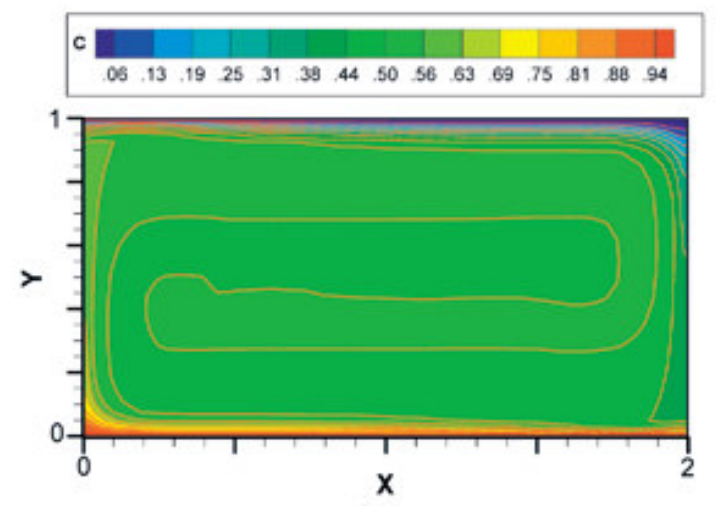

a)

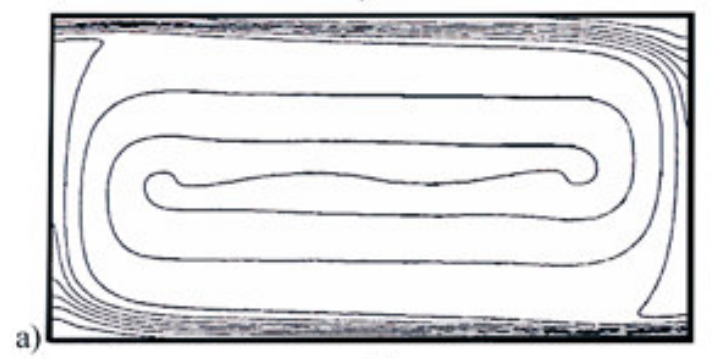

b)

Figura 6. Distribución de isoconcentraciones: a) Presente trabajo: malla $30 \times 60 ; \mathrm{t}=50 \mathrm{y}$ b) Bennacer [8]. 


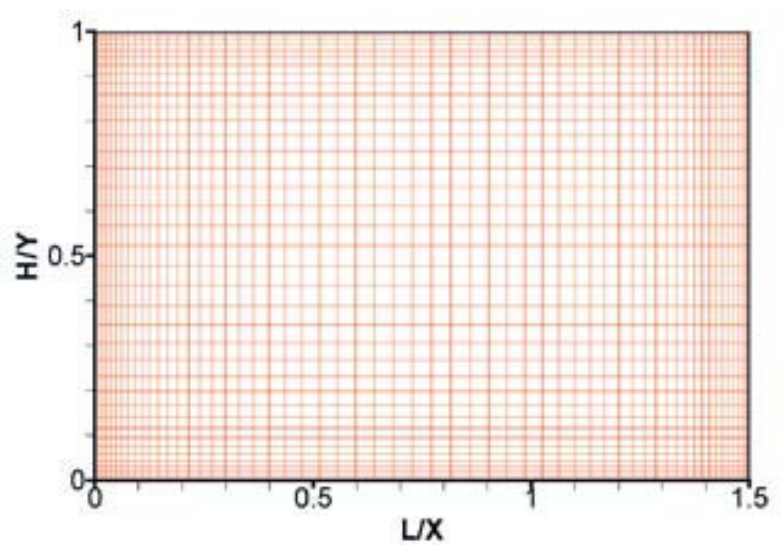

a)

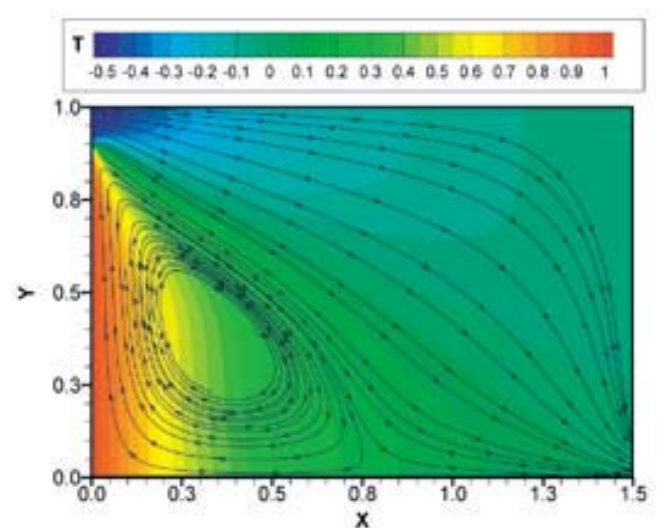

b)

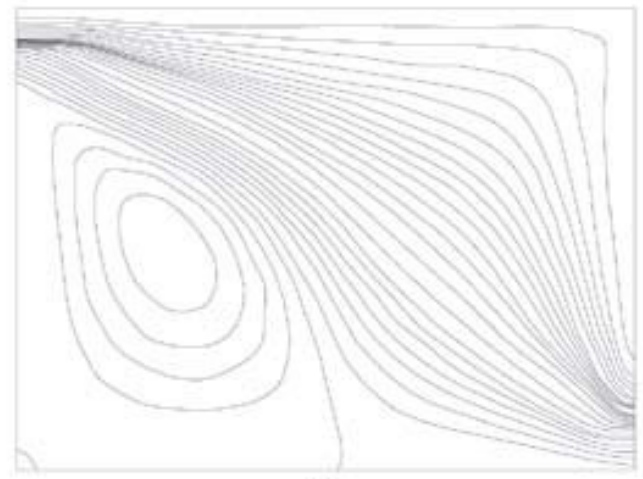

c)

Figura 7. Convección mixta: a) Malla 40x60, b) Líneas de corriente e isotermas obtenidas en el presente trabajo y c) Líneas de corrientes obtenidas Amri y Nasrallah [5].

\section{Convección mixta}

También con el objetivo de validar el modelo se generan resultados para el caso de difusión mixta isotrópica monofásica, lo cual implica considerar ingreso de masa con velocidad $\mathrm{V}$, a través de una cavidad de altura $\mathrm{d}$ sobre el extremo superior izquierdo de la pared vertical $x=0$. El egreso de masa se realiza a través de una cavidad de altura d sobre el extremo inferior derecho de la pared vertical en $\mathrm{x}=\mathrm{L}$. Resultados obtenidos para los adimensionales definidos en la tabla 3, considerando una malla no uniforme de 40x60 del tipo cosenoidal, son mostrados en las figuras (7-10).

Los resultados líneas de corriente y isotemperaturas, cuando son comparados con los obtenidos por Amri y Nasrallah [5], resultaron ser muy similares cualitativamente, pero presentan diferencias cuantitativas en la distribución de temperaturas. Para mayor detalle, las figuras (9 y 10) muestran las componentes de las velocidades cartesianas U y V, a través de estas se pueden apreciar entre otras las direcciones preferenciales de velocidad.
Tabla 3. Adimensionales para convección mixta.

\begin{tabular}{|c|c|c|c|}
\hline $\operatorname{Pr}$ & $\mathrm{Ri}$ & $\mathrm{Re}$ & $\mathrm{A}$ \\
\hline 0.7 & 0.1 & 100 & 1.5 \\
\hline
\end{tabular}

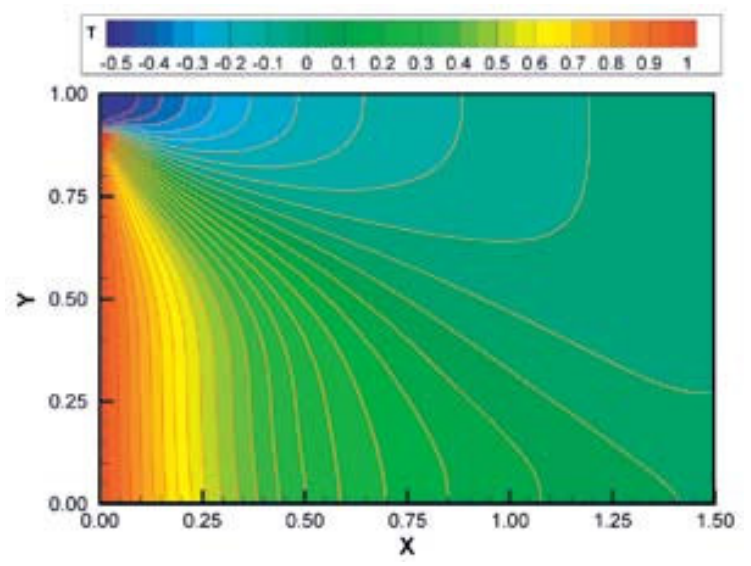

a) Obtenida en el presente trabajo.

Figura 8. Isotermas para convección mixta. (Continúa) 


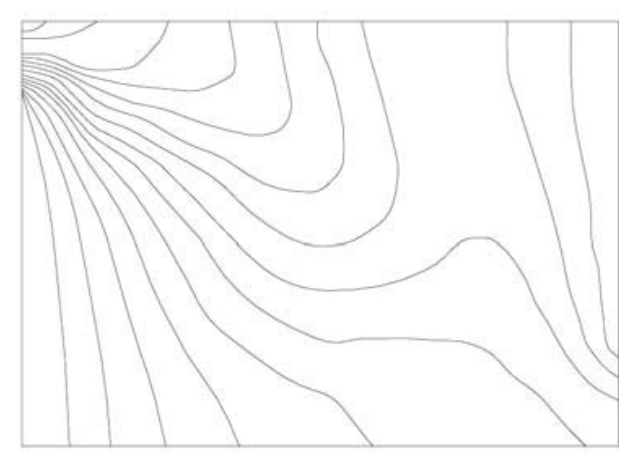

b) Obtenidas por Amri y Nasrallah.

Figura 8. Isotermas para convección mixta [5].

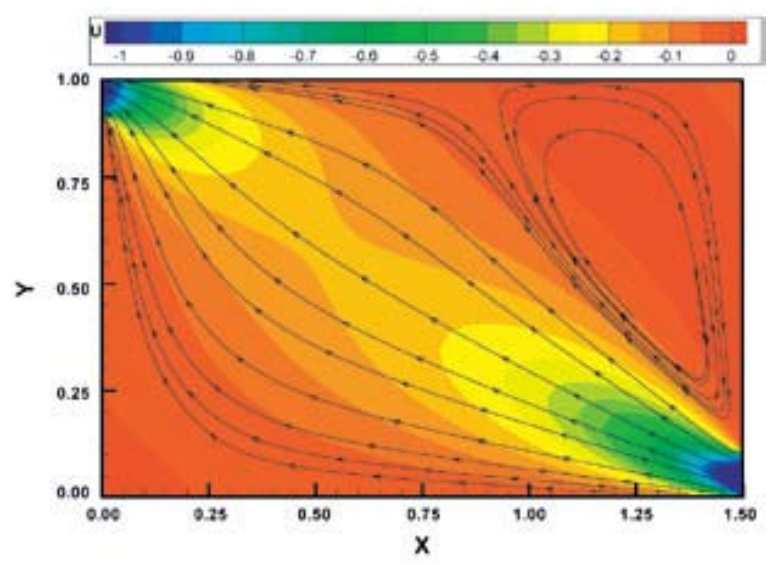

Figura 9. Componente velocidad horizontal U.

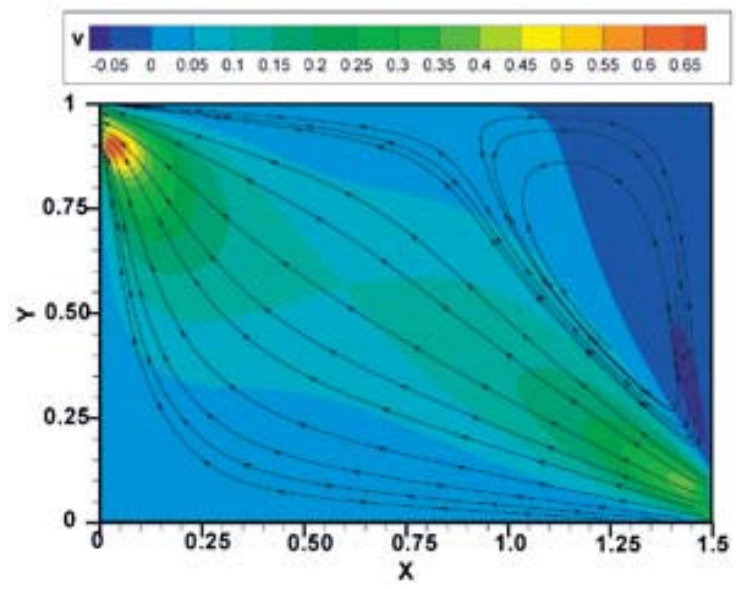

Figura 10. Componente vertical de velocidad V.

\section{Convección mixta conjugada con difusión doble}

Se plantea el problema físico de convección forzada y natural de un flujo bidimensional transiente en una cavidad rectangular acoplada con difusión doble de calor y masa como la descrita en la figura 1. Se modelan los efectos de cambios en la densidad debido a las variaciones de temperatura y concentraciones de acuerdo con la formulación de Oberdeck-Boussinesq para un fluido newtoniano-incompresible. Las condiciones de contorno son de flujo de calor en las paredes verticales y flujo de concentración en las paredes horizontales, siendo las paredes verticales y horizontales impermeables y adiabáticas respectivamente. Las condiciones iniciales y de contorno son las descritas por las ecuaciones (12) a (13).

La malla usada en las modelaciones son del tipo mostrada en la figura 11, la cual presenta estrechamiento cosenoidal hacia las paredes. Este tipo de malla ha mostrado ser consistente con los análisis de consistencia realizados en los problemas asintóticos mostrados anteriormente.

Para efectos de determinar el paso adimensional discreto de integración $\left(\mathrm{dt}^{*}\right)$ se realizaron diversas variaciones de este parámetro cuyos resultados son mostrados en las figuras 12-15. En ellas se puede observar que para $\mathrm{dt}^{*}=0.5$ se tienen resultados convergentes para el estado permanente. También se observa que los valores de concentración pueden ser modelados con un dt* sustantivamente mayor.

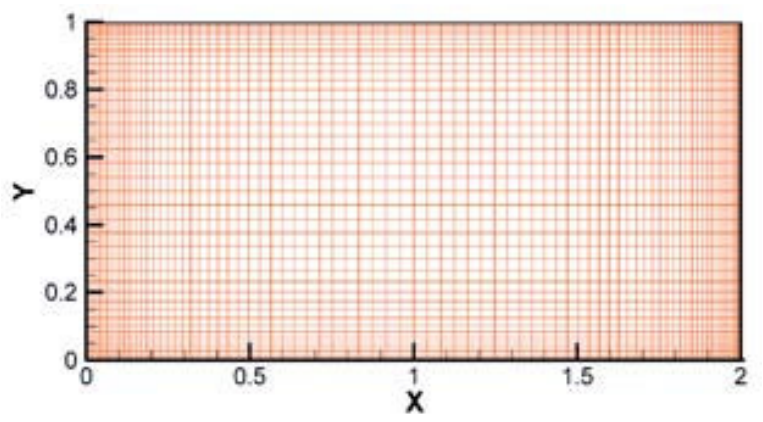

Figura 11. Malla 40x80 usada en simulación de convección mixta conjugada con difusión doble. 


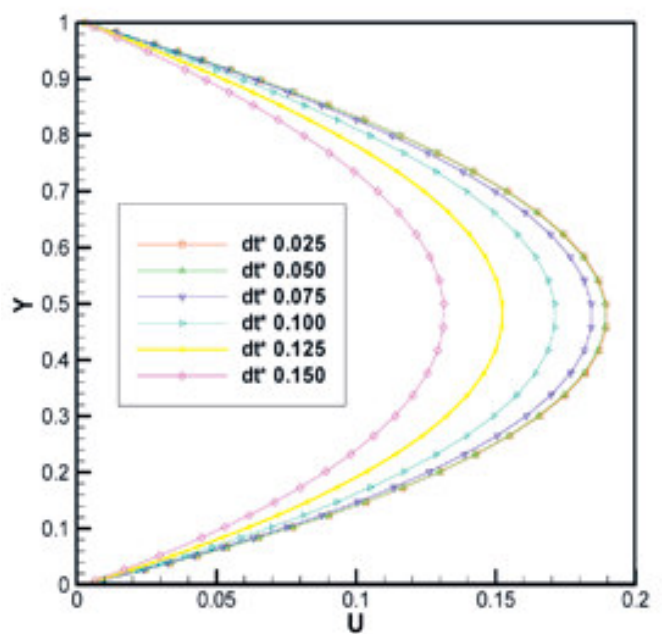

Figura 12. Componentes de velocidad $\mathrm{U}$ en $\mathrm{X}=1(\mathrm{Re}=10$, $\mathrm{N}=2.5, \operatorname{Pr}=7.0, \mathrm{Gr}_{\mathrm{T}}=10^{4}$, Le=100, $\mathrm{A}=2$ ).

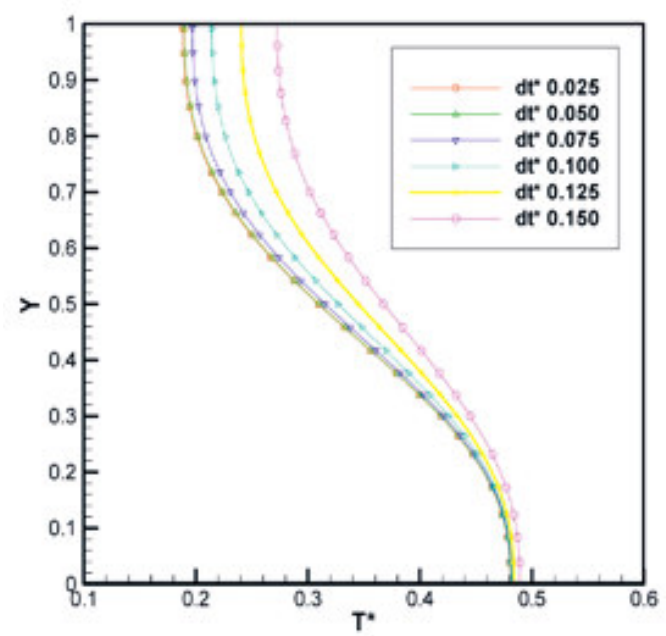

Figura 14. Temperaturas en $\mathrm{X}=1(\mathrm{Re}=10, \mathrm{~N}=2.5, \mathrm{Pr}=7.0$, $\left.\mathrm{Gr}_{\mathrm{T}}=10^{4}, \mathrm{Le}=100, \mathrm{~A}=2\right)$.

En cuanto a las distribuciones de flujo, calor y concentración, son mostradas en las figuras 16-21. El flujo es mostrado a través de sus componentes cartesianas adimensionales $\mathrm{U}$ y $\mathrm{V}$ en las figuras 16 y 17 , respectivamente. Se observa un claro dominio de la componente longitudinal $\mathrm{U}$, debido a la relación de aspecto $2 / 1$, resultado de la trayectoria natural del flujo a través de la diagonal de la cavidad con marcada recirculación en la contradiagonal producto de expansiones y contracciones en la entrada y salida de la cavidad. De forma similar son mostradas

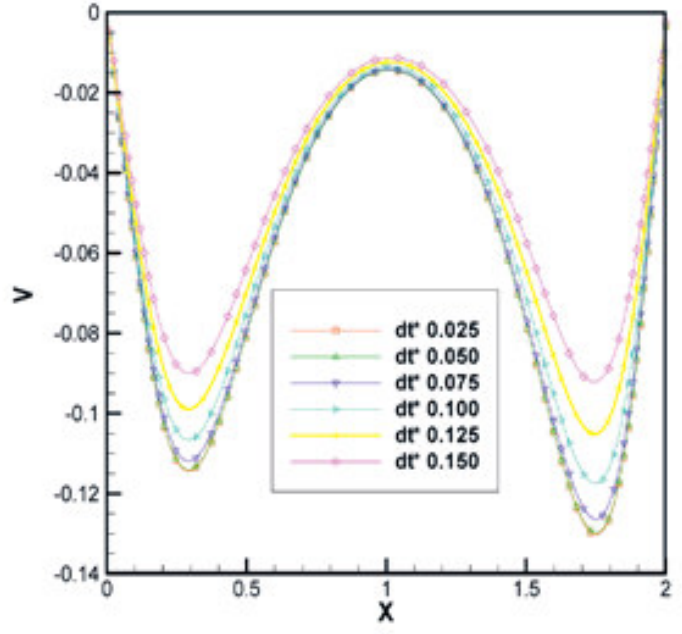

Figura 13. Componentes de velocidad $\mathrm{V}$ en $\mathrm{Y}=0.5(\mathrm{Re}=10$, $\mathrm{N}=2.5, \operatorname{Pr}=7.0, \mathrm{Gr}_{\mathrm{T}}=10^{4}$, Le=100, $\mathrm{A}=2$ ).

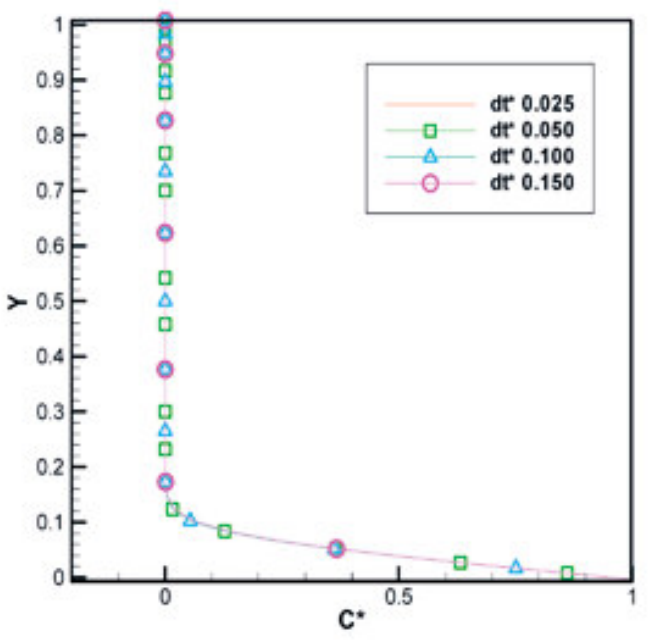

Figura 15. Concentraciones en $X=1(R e=10, N=2.5$, $\left.\operatorname{Pr}=7.0, \mathrm{Gr}_{\mathrm{T}}=10^{4}, \mathrm{Le}=100, \mathrm{~A}=2\right)$.

las distribuciones de temperatura y concentración en las figura 18 y 19. La figura 18 muestra una distribución amplia de temperaturas y se observan paralelas en las paredes verticales y normales en las horizontales, lo que demuestra una correcta modelación de las condiciones de contorno. En cuanto a las concentraciones mostradas en la figura 19, se observan bastante concentradas sobre la pared horizontal inferior de alta concentración y una difusión muy leve hacia la pared horizontal superior de baja concentración. 

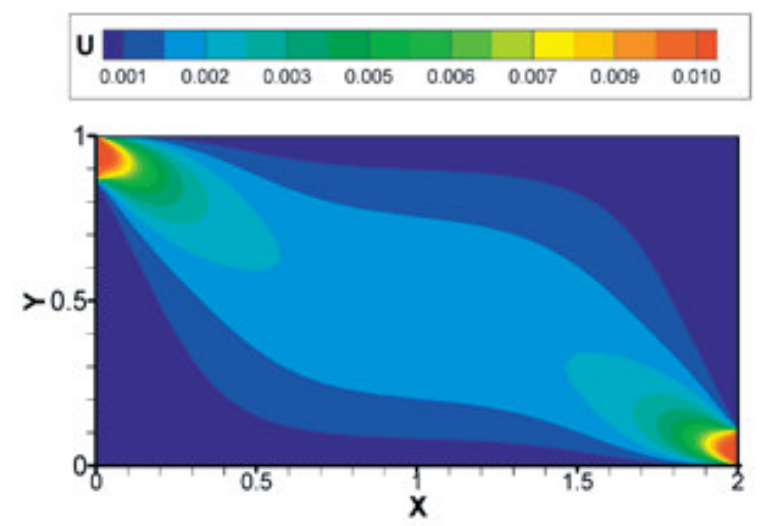

Figura 16. Distribución de velocidad $\mathrm{U}(\mathrm{Re}=10, \mathrm{~N}=2.5$, $\operatorname{Pr}=7.0, \mathrm{Gr}_{\mathrm{T}}=10^{4}, \mathrm{Le}=100, \mathrm{~A}=2$ ).

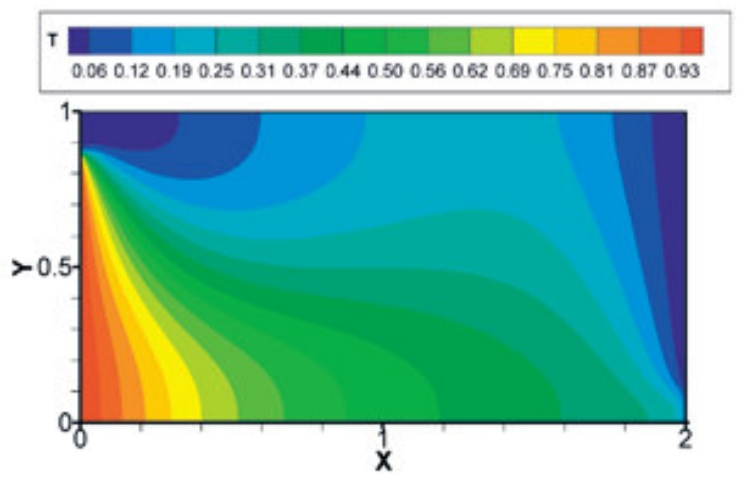

Figura 18. Isotermas $\left(\operatorname{Re}=10, \mathrm{~N}=2.5, \operatorname{Pr}=7.0, \mathrm{Gr}_{\mathrm{T}}=10^{4}\right.$, $\mathrm{Le}=100, \mathrm{~A}=2$ ).

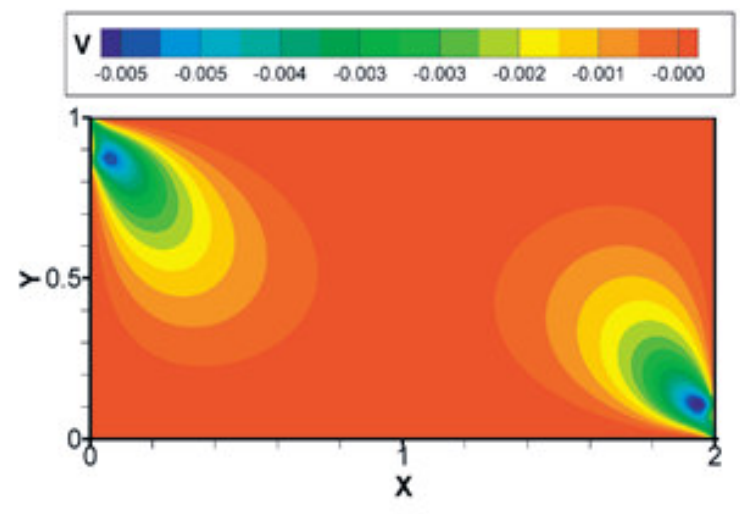

Figura 17. Distribución de velocidad $\mathrm{V}(\mathrm{Re}=10, \mathrm{~N}=2.5$, $\left.\operatorname{Pr}=7.0, \mathrm{Gr}_{\mathrm{T}}=10^{4}, \mathrm{Le}=100, \mathrm{~A}=2\right)$.

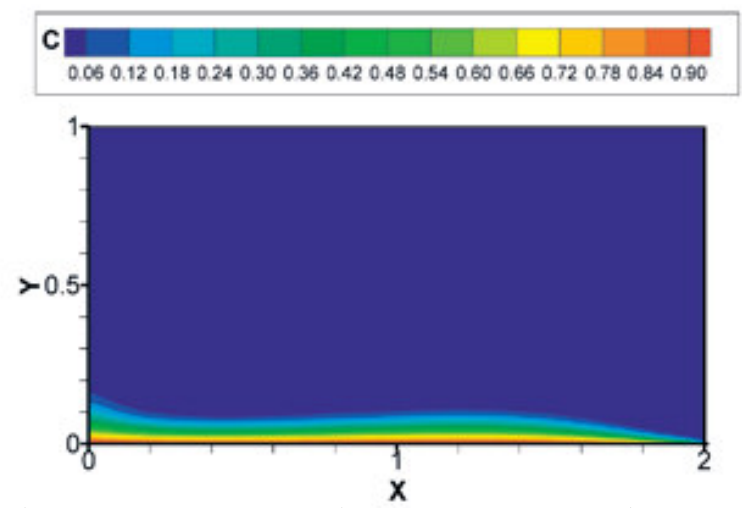

Figura 19. Isoconcentraciones $(\mathrm{Re}=10, \mathrm{~N}=2.5, \operatorname{Pr}=7.0$, $\mathrm{Gr}_{\mathrm{T}}=10^{4}, \mathrm{Le}=100, \mathrm{~A}=2$ ).

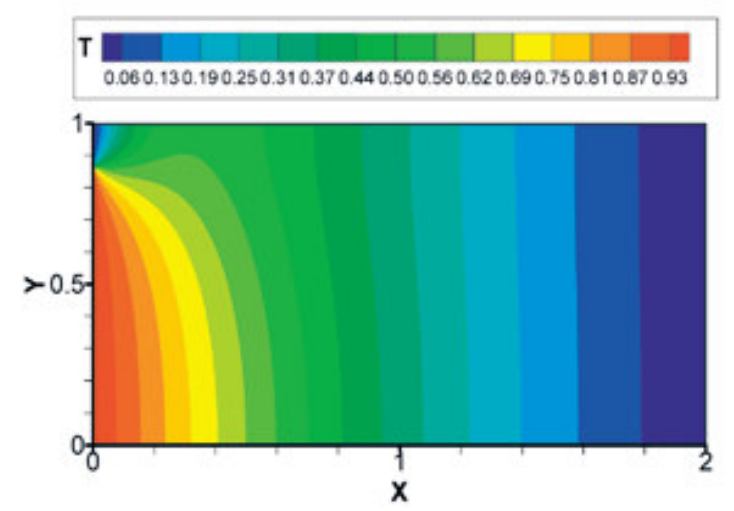

Figura 20. Isotermas $\left(\mathrm{Re}=5, \mathrm{~N}=9.0, \mathrm{Pr}=7.0, \mathrm{Gr}_{\mathrm{T}}=10^{4}, \mathrm{Le}=100, \mathrm{~A}=2\right)$.

Las figuras 20 y 21 muestran distribuciones de temperatura y concentración de una variación del número de Reynolds $(\mathrm{Re}=5)$ y relación de flotabilidad $(\mathrm{N}=9)$ de los resultados anteriores. En ellas se muestran especialmente paralelas las isoconcentraciones e isotermas, lo cual es coherente con lo mostrado por Bennacer (2001) [8] en difusión doble (obsérvese que se baja la convección forzada a la mitad del caso documentado anteriormente). 
Resultados transientes de valores medios de temperaturas y concentración para diversos números de Reynolds son mostrados en la figura 22. En estos se puede observar una sustantiva variación de la concentración conforme se acerca al estado permanente y, por otro lado, la temperatura presenta una despreciable diferencia para el mismo caso. Lo anterior es debido principalmente a la posición de las paredes de alta concentración y temperatura y su correspondiente alta y baja difusión de calor y masa, respectivamente.

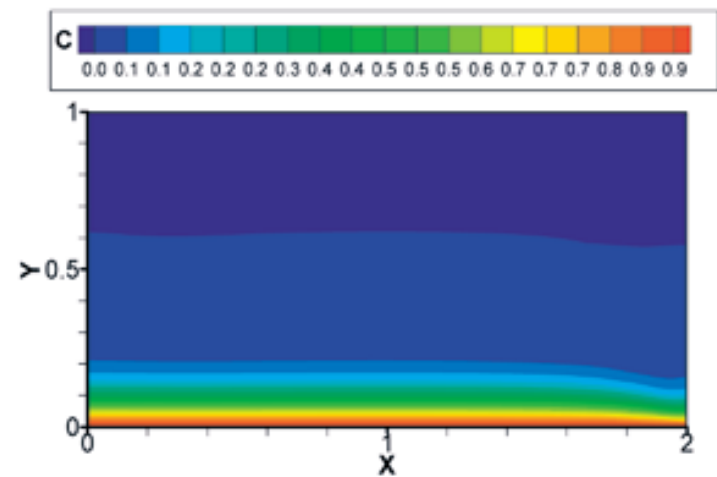

Figura 21. Isoconcentraciones $(\mathrm{Re}=5, \mathrm{~N}=9.0, \mathrm{Pr}=7.0$, $\mathrm{Gr}_{\mathrm{T}}=10^{4}, \mathrm{Le}=100, \mathrm{~A}=2$ ).

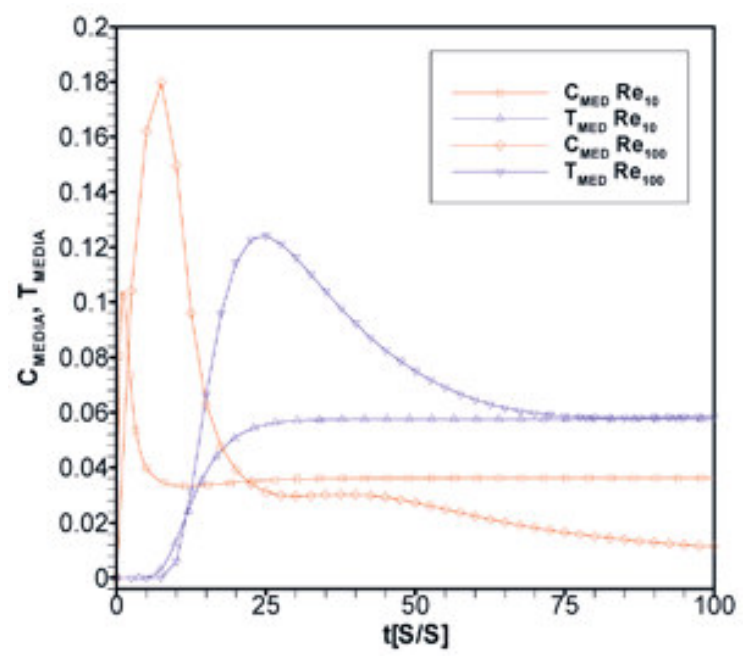

Figura 22. Valores medios de concentración y temperatura en $\mathrm{X}=2\left(\mathrm{~N}=2.5, \mathrm{Pr}=7.0, \mathrm{Gr}_{\mathrm{T}}=10^{4}, \mathrm{Le}=100\right.$, $A=2$ ).

Las figuras 23 y 24 presentan las características convectivas en función de la posición y del número de Reynold de las paredes de alta temperatura y concentración respectivamente, donde se aprecia una marcada no uniformidad conforme la cercanía a la entrada y salida de flujo para el número de Nusselt y Sherwood, respectivamente.

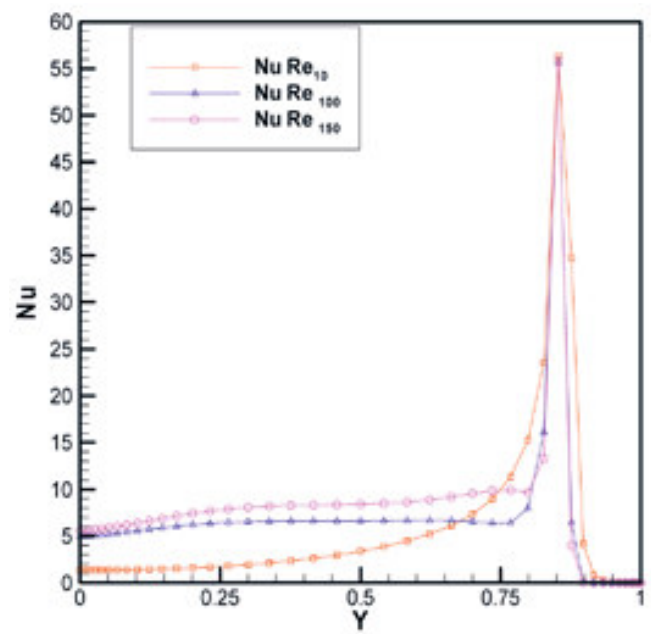

Figura 23. Número de Nusselt en $X=0(\mathrm{~N}=2.5, \mathrm{Pr}=7.0$, $\mathrm{Gr}_{\mathrm{T}}=10^{4}, \mathrm{Le}=100, \mathrm{~A}=2$ ).

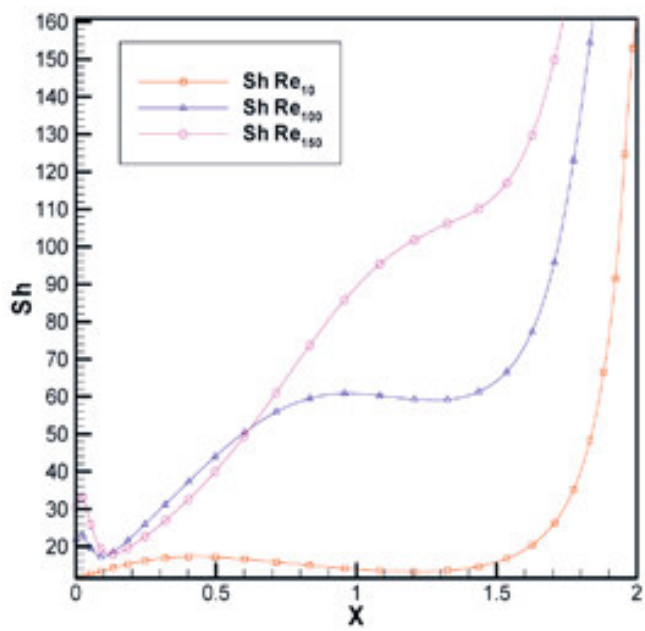

Figura 24. Número de Sherwood en $\mathrm{Y}=0(\mathrm{~N}=2.5, \mathrm{Pr}=7.0$, $\mathrm{Gr}_{\mathrm{T}}=10^{4}, \mathrm{Le}=100, \mathrm{~A}=2$ ).

La figura 25 muestra la marcada diferencia de la concentración media para un par de variaciones de número de flotación y número de Reynolds. Finalmente, la figura 26 muestra la convergencia del algoritmo computacional para las diversas variables independientes en función del número de iteraciones. Se observa una alta tasa de convergencia para todas las variables, lo que denota una buena estabilidad del algoritmo computacional. 


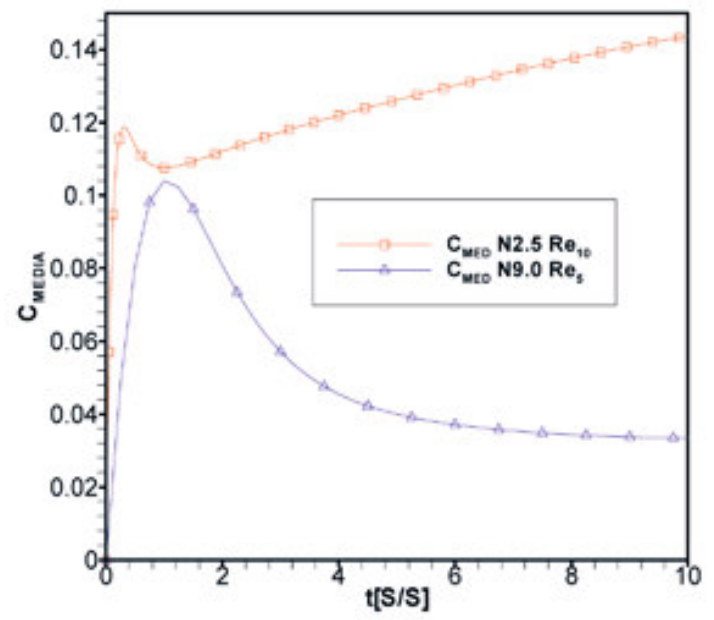

Figura 25. Concentración media en $\mathrm{X}=2\left(\mathrm{Pr}=7.0, \mathrm{Gr}_{\mathrm{T}}=10^{4}\right.$, $\mathrm{Le}=100, \mathrm{~A}=2$ ).

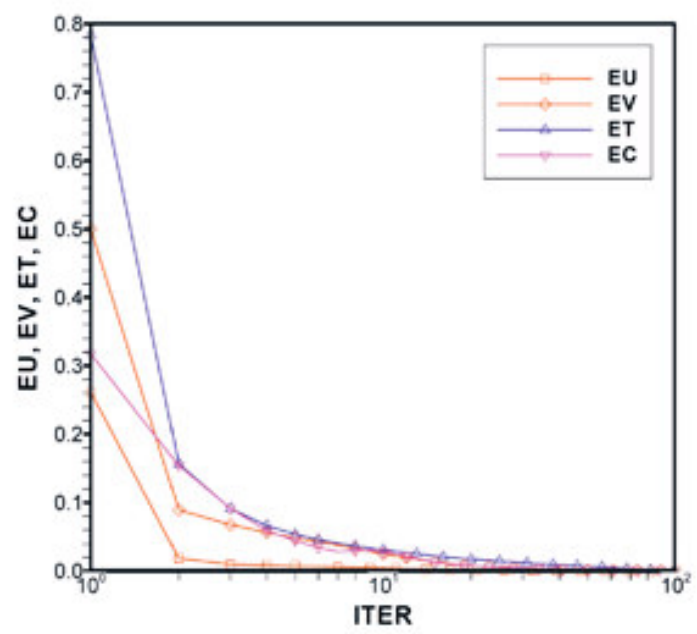

Figura 26. Máxima variación relativa EU, EV, ET y EC en la determinación de $\mathrm{U}, \mathrm{V}, \mathrm{T}$ y $\mathrm{C}$ respectivamente $\left(\mathrm{Re}=10, \mathrm{~N}=2.5, \mathrm{Pr}=7.0, \mathrm{Gr}_{\mathrm{T}}=10^{4}, \mathrm{Le}=100\right.$, $\mathrm{A}=2$ ).

\section{CONCLUSIONES}

Se ha mostrado un análisis numérico que muestra en términos generales un modelo que permite una simulación efectiva del fenómeno de convección mixta asociada a difusión de calor y masa al interior de un dominio rectangular. Esto fue mostrado para: $10 \leq \operatorname{Re} \leq 150$, $\mathrm{N}=2.5, \mathrm{Pr}=7.0, \mathrm{Gr}_{\mathrm{T}}=10^{4}, \mathrm{Le}=100, \mathrm{~A}=2$.

El modelo es validado para el transporte de calor y masa a través de comparación de resultados de problemas similares abordados en la literatura especializada: Difusión doble y convección mixta. Lo anterior permite una efectiva modelación del fenómeno conjugado de convección mixta (natural y forzada) con difusión doble.

Resultados originales mostrados caracterizan el problema planteado, observándose los padrones de flujo, distribuciones de calor y masa y las características de convección de las paredes a través de sus adimensionales característicos. Dichos resultados indican que el fenómeno conjugado sólo es apreciable (alteración del flujo) para flujos mixtos caracterizados por un bajo número de Reynolds.

\section{AGRADECIMIENTOS}

Se agradece a Conicyt y a la Universidad del Bío-Bío por el financiamiento de los proyectos FONDECYT $\mathrm{N}^{\circ} 1030209$ y FPI No 0152112 , respectivamente.

\section{REFERENCIAS}

[1] A.F. Emery and J.W. Lee. "The effects of property variations on natural convection in square enclosure". Journal of Heat Transfer. Vol. 121, pp. 57-61. 1999.

[2] A.C. Bytas and I. Pop. "Free convection in oblique enclosures filled with a porous medium". International Journal of Heat and Mass Transfer. Vol. 42, pp. 1047-1057. 1999.

[3] R.J. Yang and L.M. Fu. "Thermal and flow analysis of a heated electronic component". International Journal of Heat and Mass Transfer. Vol. 44, pp. 2261-2275. 2001.

[4] Shun-Shing Lee. "Finite element solutions of laminar and turbulent flows with forced and mixed convection in an air-cooled room". Num. Heat Transfer Part A. Vol. 31, pp. 529-550. 1997.

[5] Amri and S. Ben Nasrallah. "Control volume finite numerical simulation of mixed convection in an air-cooled cavity". Numerical Heat Transfer. Part A. Vol. 36, pp. 615-637. 1999.

[6] A.J. Chamkha and H. Al-Naser. "Double-diffusive convection in an inclined porous enclosure with opposing temperature and concentration gradients". International Journal Therm. Sci. Vol. 40, pp. 227-244. 2001. 
[7] A.A. Mohamad and R. Bennacer. "Double diffusion, natural convection in an enclosure filled with saturated porous medium subjected to cross gradients; stably stratified fluid". International Journal of Heat and Mass Transfer. Vol. 45, pp. 3725-3740. 2002.

[8] R. Bennacer, A. Mohamad and D. Akrour. "Transient natural flow in an enclosure with temperature and vertical solutal gradients". Int. J. of Therm. Sci. Vol. 40, pp. 899-910. 2001.

[9] J.R. Khan, Culham and M.M. Yovanovich. "Fluid flow around and heat transfer from an infinite circular cylinder". Journal of Heat Transfer. Vol. $127 \mathrm{~N}^{\circ}$ 7. W.A, pp: 785-790. 2005.

[10] J. Buongiorno. "Convective transport in nanofluids". Journal of Heat Transfer. Vol. $128 \mathrm{~N}^{\circ} 3$, pp. 240250. 2006.

[11] A. Haji-Sheikh. "Fully developed heat Transfer to tluid flow in rectangular passages filled with porous materials". Journal of Heat Transfer. Vol. $128 \mathrm{~N}^{\circ}$ 6, pp. 550-556. 2006.
[12] K. Hooman and Ali A. Merrikh. "Analytical solution of forced convection in a duct of rectangular cross section saturated by a porous medium". Journal of Heat Transfer. Vol. 128 No 6, pp. 596-600. 2006.

[13] E.P.F. de Pina and M. S. Carvalho. "Threedimensional flow of a newtonian liquid through an annular space with axially varying eccentricity". Journal of Fluids Engineering. Vol. $128 \mathrm{~N}^{\mathrm{o}} 2$, pp. 223-231. 2006.

[14] S.V. Patankar. "Numerical heat transfer and fluid flow". Hemisphere Publishing Corporation, Washington, DC. 1980.

[15] J.F. Thompson, Z.U.A Warsi and C.W. Mastin. "Numerical grid generation". Elsevier Science. Publishing. 1985.

[16] C. Hirsch. "Numerical computation of internal and external flows". Vol. 2. John Wiley \& Sons, Inc. New York. 1990.

[17] G.R. Maliska. "Transferencia de calor y mecánica de fluidos computacional". Livros Técnicos y Científicos Editora S.A. R.J. Brasil. 1995. 